\title{
Physical therapy for airway clearance improves cardiac autonomic modulation in children with acute bronchiolitis
}

\author{
Cynthia P. Jacinto, Ada C. Gastaldi, Daniela Y. Aguiar, \\ Karina D. Maida, Hugo C. D. Souza
}

\begin{abstract}
Background: The effects of physical therapy on heart rate variability (HRV), especially in children, are still inconclusive. Objective: We investigated the effects of conventional physical therapy (CPT) for airway clearance and nasotracheal suction on the HRV of pediatric patients with acute bronchiolitis. Method: 24 children were divided into two groups: control group (CG, $\mathrm{n}=12$ ) without respiratory diseases and acute bronchiolitis group $(\mathrm{BG}, \mathrm{n}=12)$. The heart rate was recorded in the BG at four different moments: basal recording (30 minutes), 5 minutes after the CPT (10 minutes), 5 minutes after nasotracheal suction (10 minutes), and 40 minutes after nasotracheal suction (30 minutes). The CG was subjected to the same protocol, except for nasotracheal suction. To assess the HRV, we used spectrum analysis, which decomposes the heart rate oscillations into frequency bands: low frequency ( $\mathrm{LF}=0.04-0.15 \mathrm{~Hz})$, which corresponds mainly to sympathetic modulation; and high frequency $(\mathrm{HF}=0.15-1.2 \mathrm{~Hz})$, corresponding to vagal modulation. Results: Under baseline conditions, the BG showed higher values in LF oscillations, lower values in HF oscillations, and increased LF/ HF ratio when compared to the CG. After CPT, the values for HRV in the BG were similar to those observed in the CG during basal recording. Five minutes after nasotracheal suction, the BG showed a decrease in LF and HF oscillations; however, after 40 minutes, the values were similar to those observed after application of CPT. Conclusions: The CPT and nasotracheal suction, both used for airway clearance, promote improvement in autonomic modulation of HRV in children with acute bronchiolitis.
\end{abstract}

Keywords: physical therapy; cardiac autonomic modulation; respiratory diseases; pediatric patients. Clinical Trials Identifier: NCT01354561

\section{HOW TO CITE THIS ARTICLE}

Jacinto CP, Gastaldi AC, Aguiar DY, Maida KD, Souza HCD. Physical therapy for airway clearance improves cardiac autonomic modulation in children with acute bronchiolitis. Braz J Phys Ther. 2013 Nov-Dec; 17(6):533-540. http://dx.doi.org/10.1590/ S1413-35552012005000120

\section{Introduction}

Respiratory affections are the main factors of morbidity and mortality in children given that newborns have peculiar aspects involving the respiratory system that can easily lead to respiratory failure $^{1}$ and acute viral bronchiolitis is a respiratory infection frequently seen in children younger than 2 years old. The objective of the use of respiratory physical therapy in these children is to assist in the removal of secretions and, consequently, improve oxygenation. Because these pediatric patients may not cooperate, we can use passive techniques, such as percussion, vibration, and postural drainage also known as conventional physical therapy (CPT), prolonged slow expiration (PSE), and expiratory flow increase technique followed (or not) by nasotracheal suction. However, the results for efficacy and side effects are controversial ${ }^{2-4}$.
In fact, the objective of respiratory physical therapy is a decrease in the length of hospital stay, thus decreasing hospital-related physical, psychological, and social stress ${ }^{5}$. Pediatric inpatients may present significant alterations in cardiovascular autonomic control, since constant oscillations in the cardiac and hemodynamic parameters are observed. However, there are very few studies correlating heart rate variability (HRV) and respiratory pathological conditions in pediatric inpatients either before or after respiratory physical therapy procedures, such as application of CPT.

The study of HRV is a method allowing non-invasive and selective evaluation of the changes in cardiac autonomic modulation. HRV oscillations in the low frequency band (LF: $0.04-0.15 \mathrm{~Hz}$ ) in awake humans have been 
associated with sympathetic and parasympathetic modulation, whereas oscillations in the high frequency band (HF: $0.15-0.5 \mathrm{~Hz}$ ) have been associated only with parasympathetic (vagal) modulation ${ }^{6}$.

The use of HRV analysis in several clinical situations has provided important information and serves as an evaluation instrument for a better understanding of the influence of the autonomic nervous system in various physiopathological conditions $^{6}$. With regard to the applicability of HRV analysis in pediatric populations, it is known that the maturity progression of the sympathetic and vagal divisions is accompanied by a growing increase in autonomic modulation over the pre- and post-natal periods ${ }^{7}$. In turn, a few studies have addressed the association between pathological pediatric conditions and changes in the cardiac autonomic modulation, consequently, HRV.

In light of these facts, our hypothesis is that pediatric inpatients with acute bronchiolitis may have impairments in HRV, and respiratory physical therapy can play a positive role in improving the cardiac autonomic parameters. In this sense, it is possible that HRV analysis, a relatively simple and non-invasive instrument, can reflect the effects of respiratory physical therapy on autonomic modulation. Therefore, the objective of the present study was to assess the effects of respiratory conventional physical therapy (CPT) and nasotracheal suction on autonomic modulation in pediatric inpatients with acute viral bronchiolitis.

\section{Method}

This is an interventional controlled study that included 24 term-born children into two groups: control group (CG), consisting of children with normal respiratory condition $(\mathrm{N}=12 ; 7$ females and 5 males), and bronchiolitis group (BG), consisting of hospitalized children with acute viral bronchiolitis ( $\mathrm{N}=12 ; 7$ females and 5 males) and mean hospital stay length of $13 \pm 2$ days. The age ranged from 2 to 11 months (with a mean age of $6 \pm 2$ months).

Both groups were submitted to a physical therapy treatment protocol and measurements, and the multidisciplinary medical team was instructed on the collection procedures. The children's caregivers were also informed about the procedures and the objective of this study, with all of them signing an informed consent approved by the Research Ethics Committee of the Clinical Hospital of the School of Medicine of Ribeirão Preto, Universidade de São Paulo (USP), Ribeirão Preto, SP, Brazil (No.13464/2006).

Personal and clinical data were obtained from medical records. Heart rate (HR), respiratory rate $(\mathrm{RR})$, and oxygen saturation $\left(\mathrm{SpO}_{2}\right)$ were measured by one of the investigators (Cynthia Pelegrino Jacinto, CJP). The following exclusion criteria were adopted in the present study: age above 12 months, presence of cardiovascular disease or chronic respiratory disease, prescription of vasoactive drugs or sedatives, invasive mechanical ventilation, and children on contact isolation.

\section{Experimental protocol}

Physical therapy techniques: Respiratory physical therapy using CPT was applied by the same physical therapist and conducted according to auscultation, patient tolerance, and clinical evaluation. The CPT protocol included thoracic percussion, manual vibration, postural drainage, and cough stimulation. In the BG, CPT was followed by nasotracheal suction, if necessary. The CG received the same physical therapy treatment, except nasotracheal suction, which was not performed due to ethical reasons.

Heart Rate Recording: The collection procedure from the $\mathrm{CG}$ and $\mathrm{BG}$ was performed using a heart rate monitor (Polar ${ }^{\circledR}$ - model S810i) that received the heart rate (HR) signals on a beat-by-beat basis by means of a transmission belt made of elastic material $(3 \mathrm{~cm}$ width) containing embedded electrodes connected to a frequency transmitter. The transmission belt was positioned around the child's chest next to the xiphoid process without causing pain or discomfort. HR was recorded with the children in dorsal decubitus at 30-degree elevation at four different moments in sequence: basal recording for 30 minutes before performing the respiratory CPT; 5 minutes after CPT (10 minutes); 5 minutes after nasotracheal suction (10 minutes); and 40 minutes after nasotracheal suction (30 minutes).

Analysis of the Heart Rate Variability. The HRV analysis was performed using interval time series on a beat-by-beat basis obtained from the frequency records, transferred to computer through an interface (Polar IR Interface ${ }^{\mathrm{TM}}$ ) using specific software (Polar Precision Performance SW ${ }^{\circledR}$ ). The resulting time series were divided into continuous segments of 200 beats per minute, with $50 \%$ superposition between the adjacent segments. After calculating means and variance for each segment, these values were submitted to spectral analysis through the autoregressive method as described in the literature ${ }^{8,6}$. The oscillatory components existing in the stationary 
segments of the beat-by-beat interval time series were modeled by using the Levinson-Durbin recursion, and the method was chosen according to Akaike's criteria ${ }^{8}$. This procedure allows the frequency axis and power of each relevant oscillatory component in the time series to be automatically quantified. The oscillatory components were classified as follows: very-low frequency (VLF: 0.001 to $0.04 \mathrm{~Hz}$ ), low frequency (LF: 0.04 to $0.15 \mathrm{~Hz}$ ), and high frequency (HF: 0.15 to $1.2 \mathrm{~Hz}$ ). LF and HF oscillation power was also expressed in normalized units, which was obtained by calculating the ratio between band power (LF or HF) and total power after subtracting the VLF component. The normalization procedure was performed in order to minimize the total power variations in the absolute values of $\mathrm{LF}$ and $\mathrm{HF}$ components $^{8,6}$. In addition, the $\mathrm{LF} / \mathrm{HF}$ ratio was calculated to establish an index for assessment of cardiac autonomic modulation.

\section{Statistical analysis}

The sample size calculation was performed using the "GraphPad StatMate 1.01", confidence level of $95 \%$, Power $80 \%$ using the LF variable in normalized units. A sample of 12 children was suggested. All statistical tests were performed with SigmaStat 3.5 software (Systat Software Inc., San Jose, CA, USA). The Shapiro-Wilk normality test was used, and the data are expressed as mean \pm standard deviation of the mean (SD). A one-way ANOVA followed by the Student-Newman-Keuls multiple comparison test was performed to evaluate the effects of respiratory CPT and nasotracheal suction on the HRV of the CG vs. BG. Student's t test was used for the comparisons between groups before respiratory ACT. The differences were considered significant at $\mathrm{P}<0.05$.

\section{- Results}

Table 1 shows mean values for $\mathrm{HR}, \mathrm{RR}$, and $\mathrm{SpO}_{2}$ at baseline and after respiratory $\mathrm{CPT}$ application. The results showed that the $\mathrm{BG}$ had higher $\mathrm{HR}$ and lower $\mathrm{SpO}_{2}$ than the CG before respiratory CPT. However, after the respiratory CPT the values were similar.

Table 2 shows the baseline values of the HRV in the frequency domain evaluated by spectral analysis before and after the respiratory CPT. The BG showed lower RRi values before the respiratory CPT, greater sympathetic autonomic influence, and lower vagal influence characterized by major oscillations in the LF band and minor oscillations in the HF band in absolute and normalized units when compared to the CG. Consequently, the BG showed a higher LF/HF
Table 1. Values for heart rate (HR), respiratory rate (RR), and oxygen saturation $\left(\mathrm{SpO}_{2}\right)$ obtained from the control group (CG) and the bronchiolitis group (BG) before (pre) and after (post) respiratory conventional physical therapy (CPT).

\begin{tabular}{ccccc}
\hline & \multicolumn{2}{c}{ Control Group } & \multicolumn{2}{c}{ Bronchiolitis Group } \\
& Pre-CPT & Post-CPT & Pre-CPT & Post-CPT \\
$\mathrm{HR}$ & $143 \pm 9$ & $142 \pm 8$ & $153 \pm 14^{*+}$ & $149 \pm 10$ \\
$\mathrm{RR}$ & $42 \pm 5$ & $38 \pm 6$ & $44 \pm 6$ & $39 \pm 7$ \\
$\mathrm{SpO}_{2}$ & $98 \pm 2$ & $97 \pm 2$ & $95 \pm 3^{*}$ & $96 \pm 2$ \\
\hline
\end{tabular}

All values expressed as mean $\pm \mathrm{SD}$. $* \mathrm{P}<0.05$ vs. $\mathrm{CG}$ before conventional physical therapy (pre-CPT); ${ }^{+} \mathrm{P}<0.05$ vs. $\mathrm{CG}$ after conventional physical therapy (post-CPT).

ratio compared to the $\mathrm{CG}$ indicating an exaggerated predominance of cardiac sympathetic modulation in relation to the CG. However, after respiratory CPT, the BG had values similar to the CG suggesting an improvement in cardiac autonomic modulation.

Table 3 shows the behavior of HRV at four different sequential moments: basal recording (30 minutes preCPT); 5 minutes after CPT application (10-minute record post-CPT); 5 minutes after nasotracheal suction (10-minute record); and 40 minutes after nasotracheal suction (30-minute record). Following the application of respiratory CPT, there was a decrease in LF oscillations and an increase in HF oscillations compared to baseline values in absolute and normalized units as shown previously (Table 2). In turn, 5 minutes after nasotracheal suction, there were reductions in overall variance and in LF and HF oscillations (compared to those values after CPT) in absolute and normalized units resulting in a significant increase in the LF/HF ratio. On the other hand, 40 minutes after nasotracheal suction (final record), variability patterns similar to those observed after respiratory CPT (post-CPT) application were observed.

\section{Discussion}

HRV analysis has been studied by several researchers in the last decades. This interest has increased because HRV analysis allows non-invasive and selective evaluation of the influence of the autonomic nervous system on the cardiovascular system. The results of this growing research on HRV, mainly the clinical studies, have demonstrated that such an assessment can be used as a hemodynamic and metabolic marker, as well as a predictive index for cardiovascular events ${ }^{6}$.

In pediatric patients, the clinical use of HRV was first described in the 1960s when a study 
Table 2. Spectral parameters of R-R intervals (RRi) calculated using spectral analysis (autoregressive method) before (pre) and after (post) conventional physical therapy (CPT) for the control group (CG) and the bronchiolitis group (BG).

\section{Control Group (CG)}

Pre-CPT Post-CPT

Baseline values
$421 \pm 28$

$293 \pm 28$

$189 \pm 20$

$81 \pm 4$

$46 \pm 9$

$19 \pm 4$

$4.3 \pm 1.2$
$424 \pm 25$

$395 \pm 35^{*+}$

$405 \pm 29$

\section{Spectral parameters}

\begin{tabular}{|c|c|c|c|c|}
\hline Variance, $\mathrm{ms}^{2}$ & $293 \pm 28$ & $319 \pm 26$ & $361 \pm 21^{*+}$ & $316 \pm 35^{\#}$ \\
\hline $\mathrm{LF}, \mathrm{ms}^{2}$ & $189 \pm 20$ & $206 \pm 16$ & $228 \pm 33^{*}$ & $187 \pm 18^{\#}$ \\
\hline LF, nu & $81 \pm 4$ & $79 \pm 5$ & $88 \pm 3 *+$ & $79 \pm 4^{\#}$ \\
\hline $\mathrm{HF}, \mathrm{ms}^{2}$ & $46 \pm 9$ & $53 \pm 14$ & $30 \pm 7^{*+}$ & $49 \pm 10^{\#}$ \\
\hline $\mathrm{HF}, \mathrm{nu}$ & $19 \pm 4$ & $21 \pm 5$ & $12 \pm 3^{*+}$ & $22 \pm 4^{\#}$ \\
\hline $\mathrm{LF} / \mathrm{HF}$ & $4.3 \pm 1.2$ & $4.2 \pm 1.3$ & $8.1 \pm 2.4^{*+}$ & $4.0 \pm 1.1^{\#}$ \\
\hline
\end{tabular}

All values expressed as mean \pm SD. RRi: R-R interval; LF: low frequency; HF: high frequency; and nu, normalized units. *P<0.05 vs. CG before conventional physical therapy (pre-CPT); ${ }^{+} \mathrm{P}<0.05$ vs. CG after conventional physical therapy (post-CPT); ${ }^{\sharp} \mathrm{P}<0.05$ vs. BG before conventional physical therapy (pre-CPT).

Table 3. Spectral parameters of RR intervals (RRi) for the bronchiolitis group (BG) at four different sequential moments: before conventional physical therapy (pre-CPT), after CPT (post-CPT), 5 minutes after nasotracheal suction (5'post-NS), and 40 minutes after nasotracheal suction (40' post-NS).

\section{Bronchiolitis Group}

$\begin{array}{lll}\text { pre-CPT } & \text { post-CPT }\end{array}$

$405 \pm 29$

$389 \pm 20$

$393 \pm 30$

RRi, sec

$395 \pm 35$

$316 \pm 35^{*}$
$187 \pm 18^{*}$
$79 \pm 4^{*}$
$49 \pm 10^{*}$
$21 \pm 4^{*}$

$4.0 \pm 1.1^{*}$

$\begin{array}{cc}132 \pm 34^{*+} & 280 \pm 51^{* \#} \\ 96 \pm 28^{*+} & 169 \pm 38^{* \#} \\ 89 \pm 4^{+} & 74 \pm 10^{* \#} \\ 12 \pm 4^{*+} & 56 \pm 19^{* \#} \\ 11 \pm 4^{+} & 26 \pm 12^{* \#} \\ 9.3 \pm 4.4^{+} & 3.5 \pm 1.3^{* \#}\end{array}$

All values expressed as mean $\pm \mathrm{SD}$. RRi: R-R interval; LF: low frequency; HF: high frequency; and nu, normalized units. *P<0.05 vs. before conventional physical therapy (pre-CPT); ${ }^{+} \mathrm{P}<0.05$ vs. after conventional physical therapy (post-CPT); ${ }^{*} \mathrm{P}<0.05$ vs. 5 minutes after nasotracheal suction ( $5^{\prime}$ post-NS).

demonstrated that fetal distress was preceded by alterations in the inter-beat intervals long before any heart rate change ${ }^{9}$, but not until the 1980s did the advent of computer techniques allow the concept of HRV analysis to be employed for evaluation of cardiovascular autonomic control ${ }^{10}$.

Several linear and non-linear methods have been applied to HRV analysis. However, spectral analysis is the most common linear method used in the frequency domain, decomposing the crucial oscillatory components into their occurrence frequencies, which allows investigation of the influence of autonomic, sympathetic (LF), and parasympathetic (HF) components on the HR modulation. Therefore, this method can be applied to individuals of all ages ${ }^{11}$.

With regard to the influence of cardiac autonomic components on cardiovascular control during fetal development and in newborns, it is know that the maturation of neuro-cardiac control increases cardio-regulatory influence on HR variability, mainly through the baroreflex system ${ }^{12}$. This neural cardiac maturation, which occurs between the $31^{\text {st }}$ and the $38^{\text {th }}$ week of gestation, is accompanied by a reduction in fetal HR. In this sense, the reduction in $\mathrm{HR}$ and $\mathrm{HRV}$ is due to a decrease in the randomized oscillatory components and to variability patterns 
coherent with the cardiac autonomic drive ${ }^{12,13}$ The importance of this maturation process on the cardiac autonomic regulation became evident in a study showing the correlation between deficient cardio-vagal development and sudden infant death syndrome ${ }^{14}$.

In contrast, the homeostasis of cardiac autonomic regulation, maintained by defined autonomic modulation patterns, can be affected by different pathological situations ${ }^{6}$. In this sense, the hypothesis raised by the present study was based on the fact that respiratory affections (acute viral bronchiolitis) in pediatric patients could provoke HRV changes resulting from the impairment of cardiac autonomic modulation and that respiratory physical therapy might promote beneficial systemic effects by restoring a more coherent autonomic influence in relation to the normality patterns.

In fact, respiratory physical therapy is mainly aimed at acting on physical and social factors affecting a given acute or chronic respiratory pathological condition. In the daily routine, the integration of a physical therapist into the multidisciplinary team speeds recovery, thus reducing the length of hospital stay. However, although respiratory physical therapy is crucial for improving the ventilatory pattern, the lack of instruments does not allow a more precise assessment of the therapy-induced systemic effects on both the cardiovascular autonomic system and homeostasis, and there are very few studies correlating pediatric respiratory pathological conditions with HRV behavior. In general, these studies were based on short-term records in which the HRV was associated with inducing tests only (respiratory procedures, postural tests, etc. ${ }^{15}$.

Accordingly, our results have shown that at baseline, that is, prior to respiratory CPT, the pediatric inpatients typically presented with increased LF and decreased HF oscillations. Despite the lack of similar studies on the pediatric population with acute viral bronchiolitis, these findings are corroborated by results obtained from adults, such as patients with COPD who showed changes in sympathetic cardiac autonomic modulation ${ }^{16,17}$. Additionally, our results have also shown that CPT intervention restored the autonomic modulation patterns of HRV in these hospitalized children by improving the cardiac autonomic balance, which was confirmed by the reduction in the $\mathrm{LF} / \mathrm{HF}$ ratio. This fact was observed after application of CPT and 40 minutes after nasotracheal suction. The improvement in autonomic modulation in both situations was supposedly due to the movement of secretions and improved respiratory function despite no significant changes in any respiratory parameter, such as respiratory rate and oxygen saturation. In the first case, the combination of CPT and cough stimulation may have improved respiratory function and, consequently, influenced the pattern of cardiac autonomic modulation. In turn, immediately after nasotracheal suction, there was a reduction in LF and HF oscillations in absolute and normalized units, possibly due to the intense stress caused by the procedure. Interestingly, this reduction in LF oscillations soon after the nasotracheal suction seems to be a paradox because of the aggressive nature of this procedure, which could increase the influence of sympathetic modulation and consequently the LF oscillations as well. However, the decrease in LF oscillations may be due to the sympathetic over-activity resulting from this extremely stressing intervention. This has also been observed in other situations, such as intense physical exercise ${ }^{18}$ or severe heart failure ${ }^{19}$. In these situations, when the physiological mechanisms are at maximum capacity in order to maintain homeostasis, the cardiovascular system may not keep its variability. In fact, the cause for reduction in LF and HF oscillations 5 minutes after nasotracheal suction is unknown, but sympathetic over-activity may be involved.

Indeed, other studies support this idea by demonstrating that hospitalized patients can exhibit symptoms such as agitation, dyspnea, cyanosis, and tachycardia, but despite the risks inherent to this procedure, the hypersecretive patients benefit from this technique ${ }^{20}$. As described above, the pattern of cardiac autonomic modulation was restored 40 minutes after nasotracheal suction, which was characterized by values of LF and HF oscillations similar to those observed following CPT application in experimental and control groups. In fact, several studies have demonstrated that the changes in HRV quickly return to baseline values following transitory perturbations induced by maneuvers such as mild exercise, administration of rapid action vasoconstrictors, and transitory coronary occlusion, whereas only more intense stimuli such as heavy exercise and administration of long-term action drugs result in a longer interval prior to the return to baseline values ${ }^{6}$. Therefore, our results showed that respiratory CPT followed (or not) by nasotracheal suction significantly improves the pattern of cardiac autonomic modulation, thus restoring values similar to those of control children with no respiratory affection.

Moreover, although our findings point to an improvement in autonomic parameters evaluated in pediatric patients with viral bronchiolitis, the 
application of respiratory CPT in this situation has been widely questioned and is the subject of discussion in the literature. In fact, the purpose of the use of respiratory physical therapy in infants with acute viral bronchiolitis is helping to remove secretions and improve oxygenation indices. However, there are arguments against respiratory physical therapy as part of routine care of these patients according to the level of bronchiolitis caused by respiratory distress, the absence of substantial proven benefits, and possible adverse effects ${ }^{21}$.

Accordingly, two review studies 2,3 that aimed to examine the efficacy of respiratory physical therapy in children under 24 months old, with acute bronchiolitis, concluded that there is not sufficiently evidence who show the benefits of physical therapy in reducing the length of hospital stay, but also concludes that the studies were insufficient to define the indication and contraindication of respiratory physical therapy. Meanwhile, a recent review of Postiaux et al. ${ }^{4}$ showed better results with conventional and new therapy techniques, especially prolonged slow expiration (PSE). However, this study concludes that further research is required to better define the indications and contraindications to CPT in patients with bronchiolitis.

A previous study ${ }^{22}$ that compared a control group with two groups submitted to CPT and expiratory flow increase technique showed lower HR values in both treatment groups, with no adverse effects attributed to CPT. Additionally, other studies have evaluated the effect of the expiratory flow increase technique in infants with bronchiolitis, two of which were multicenter studies ${ }^{23,24}$ that did not find differences in length of hospital stay. In the first ${ }^{23}$, there was a higher frequency of discomfort in the intervention group and, in the second ${ }^{25}$, there was a better clinical score in the intervention group with expiratory flow increase technique and PSE.

The PSE technique has also been used by other authors ${ }^{26-28}$. In one study, there was a lower clinical score, respiratory rate and HR for the group treated with CPT and prolonged slow expiration technique, as well as a shorter hospital stay (although not statistically significant) for the group submitted to $\mathrm{PSE}^{26}$. Another study showed shorter time evolution and reduction in hours on oxygen support in the group using vibration with PSE compared to controls ${ }^{27}$. Additionally, the results of other authors also demonstrated a better clinical score and a reduction in chest retractions in the groups treated with CPT and PSE, with improvement in oxygen saturation and bronchospasm in the group submitted only to PSE.
Finally, the results of these studies clearly show the need for other evaluation parameters more sensitive to changes promoted by respiratory physical therapy. As far as we know, this is the first study that used HRV analysis in these conditions. Accordingly, our results showed an improvement in the autonomic parameters evaluated by spectral analysis of the HRV. These findings may be related to the primary objective of the treatment of these children, which is to reduce the discomfort caused by airway secretions and reduce stress related to ventilation.

In sum, the respiratory physical therapy by means of CPT and nasotracheal suction, both used for airway clearance, promote improvement in autonomic modulation of HRV in children with acute viral bronchiolitis, suggesting that HRV analysis can be used as an indirect marker of the clinical status of patients undergoing such an intervention.

\section{Study limitations}

Some limitations regarding this study should be considered, such as long-term evaluation of the patient undergoing respiratory physical therapy and application of other interventions aimed at better interpreting the results obtained from HRV analysis (e.g.: serum dosages of catecholamine and cortisol). The choice of experimental design in which the respiratory physical therapy procedures were stratified to involve CPT and nasotracheal suction is justified by the fact that both interventions demonstrate different hemodynamic and cardiovascular parameters. Within this context, the analysis of cardiac autonomic modulation during different moments of the respiratory physical therapy has allowed us to score the HRV behavior in different procedures, thus providing another useful instrument for quantifying the risk-benefit of techniques applied to hospitalized children.

\section{Clinical implications}

The search for and use of new tools and evaluation parameters that do not promote adverse effects or compromise the patient's condition should be encouraged. In this sense, the analysis of HRV, a noninvasive and painless method, can provide valuable information about the patient's clinical status and systemic effects on the cardiovascular system, both before and after physical therapy intervention.

\section{References}

1. Latzin P, Frey U, Roiha HL, Baldwin DN, Regamey N, Strippoli MP, et al. Swiss Paediatric Respiratory Research Group. Prospectively assessed incidence, severity, and 
determinants of respiratory symptoms in the first year of life. Pediatr Pulmonol. 2007;42:41-50. PMid:17123315. http://dx.doi.org/10.1002/ppul.20542

2. Perrotta C, Ortiz Z, Roque M. Chest physiotherapy for acute bronchiolitis in paediatric patients between 0 and 24 months old. Cochrane Database Syst Rev. 2007 Jan 24;1:CD004873. PMid:17253527.

3. Perrotta C, Ortiz Z, Roque M. Chest physiotherapy for acute bronchiolitis in paediatric patients between 0 and 24 months old. Cochrane Database Syst Rev. 2012 Feb 15;2:CD004873. PMid:22336805.

4. Postiaux G, Zwaenepoel B, Louis J. Chest Physical Therapy in Acute Viral Bronchiolitis: an updated review. Respir Care. 2013 Sep;58(9):1541-5. http://dx.doi. org/10.4187/respcare.01890

5. Oberwaldner B. Physiotherapy for airway clearance in paediatrics. Eur Respir J. 2000;15:196-204. PMid:10678646. http://dx.doi.org/10.1183/09031936.0 0.15119600

6. Task Force of the European Society of Cardiology, North American Society of Pacing and Electrophysiology. Heart rate variability: standards of measurements, physiological interpretation, and clinical use. Circulation. 1996;93:104365. PMid:8598068. http://dx.doi.org/10.1161/01. CIR.93.5.1043

7. Winsley RJ, Armstrong N, Bywater K, Fawkner SG. Reliability of heart rate variability measures at rest and during light exercise in children. Br J Sports Med. 2003;37:550-2. PMid:14665601 PMCid:PMC1724706. http://dx.doi.org/10.1136/bjsm.37.6.550

8. Malliani A, Pagani M, Lombardi F, Cerutti S. Cardiovascular neural regulation explored in the frequency domain. Circulation. 1991;84:482-92. PMid:1860193. http://dx.doi.org/10.1161/01.CIR.84.2.482

9. Hon EH, Lee ST. Eletronic evaluations of the fetal heart rate patterns preceding fetal death, futher observations. Am J Obstet Gynec. 1965;87:814-26.

10. Akselrold S, Gordon D, Ubel FA, Shannon DC, Berger AC, Cohen RJ. Power spectrum analysis of heart rate fluctuation: a quantitative probe of beat-to-beat cardiovascular control. Science.1981;213:220-2. http:// dx.doi.org/10.1126/science.6166045

11. Rajendra Acharya U, Paul Joseph K, Kannathal N, Lim CM, Suri JS. Heart rate variability: a review. Med Biol Eng Comput. 2006;44:1031-51. PMid:17111118. http:// dx.doi.org/10.1007/s11517-006-0119-0

12. Mazursky JE, Birkett CL, Bedell KA, Ben-Haim AS, Segar JL. Development of baroreflex influences on heart rate variability in preterm infants. Early Hum Dev. 1998;53:3752. http://dx.doi.org/10.1016/S0378-3782(98)00038-3

13. Sahnir R, Schulze KF, Kashyap S, Ohira-Kist K, Fifer WP, Myers MM. Maturation changes in heart rate and heart rate variability in low birth weight infants. Dev Psychobiol. 2000;37:73-81. http://dx.doi.org/10.1002/10982302(200009)37:2<73::AID-DEV2>3.0.CO;2-C

14. Southall DP, Scherchtman VL, Raetz SL, Garfinkel A, Wilson AJ, Harper RM. Dynamic analysis of cardiac R-R intervals in normal infants and in infants who subsequently succumbed to the sudden infant death syndrome. Pediatr
Res. 1992;31:606-12. PMid:1635823. http://dx.doi. org/10.1203/00006450-199206000-00014

15. Massin M, Von Bernuth G. Normal ranges of heart rate variability during infancy and childhood. Pedriatr Cardiol. 1997;18:297-302. http://dx.doi.org/10.1007/ s002469900178

16. Pantoni BF, Reis MS, Martins EB, Catai AM, Costa D, Borghi-Silva A. Study on autonomic heart rate modulation at rest among elderly patients with chronic obstructive pulmonary disease. Rev Bras Fisioter. 2007;11:35-41. http://dx.doi.org/10.1590/S1413-35552007000100007

17. Stein PK, Nelson P, Rottman JN, Howard D, Ward $\mathrm{SM}$, Kleiger RE, et al. Heart rate variability reflects severity of COPD in PiZ alpha1-antitrypsin deficiency. Chest. 1998;113:327-33. PMid:9498947. http://dx.doi. org/10.1378/chest.113.2.327

18. Arai Y, Saul JP, Albrecht P, Hartley LH, Lilly LS, Cohen RJ, et al. Modulation of cardiac autonomic activity during and immediately after exercise. Am J Physiol. 1989;256(1 Pt 2):H132-41. PMid:2643348.

19. Van de Borne P, Montano N, Pagani M, Oren R, Somers VK. Absence of low-frequency variability of sympathetic nerve activity in severe heart failure. Circulation. 1997;95:1449-54. PMid:9118512. http:// dx.doi.org/10.1161/01.CIR.95.6.1449

20. Morrow BM, Argent AC. A comprehensive review of pediatric endotracheal suctioning: Effects, indications, and clinical practice. Pediatr Crit Care Med. 2008;9:46577. PMid:18679146. http://dx.doi.org/10.1097/ PCC.0b013e31818499cc

21. American Academy of Pediatrics Subcommittee on Diagnosis and Management of Bronchiolitis. Diagnosis and management of bronchiolitis. Pediatrics. 2006;118:1774-93. PMid:17015575. http://dx.doi. org/10.1542/peds.2006-2223

22. Pupin MK, Riccetto AG, Ribeiro JD, Baracat EC. Comparison of the effects that two different respiratory physical therapy techniques have on cardiorespiratory parameters in infants with acute viral bronchiolitis. J Bras Pneumol. 2009;35:860-7. PMid:19820812. http://dx.doi. org/10.1590/S1806-37132009000900007

23. Gajdos V, Katsahian S, Beydon N, Abadie V, De Pontual L, Larrar S, et al. Effectiveness of chest physiotherapy in infants hospitalized with acute bronchiolitis: a multicenter, randomized, controlled trial. PLoS Med. 2010;28;7(9):e1000345.

24. Bailleux S, Lopes D, Geoffroy A, Josse N, Labrune P, Gajdos V. What evidence for chest physiotherapy in infants hospitalized for acute viral bronchiolitis? Arch Pediatr. 2011;18:472-5. PMid:21392951. http://dx.doi. org/10.1016/j.arcped.2011.01.028

25. Rochat I, Leis P, Bouchardy M, Oberli C, Sourial H, Friedli-Burri M, et al. Chest physiotherapy using passive expiratory techniques does not reduce bronchiolitis severity: a randomised controlled trial. Eur J Pediatr. 2012;171:457-62. PMid:21927808. http://dx.doi. org/10.1007/s00431-011-1562-y

26. Postiaux G, Louis J, Labasse HC, Gerroldt J, Kotik $\mathrm{AC}$, Lemuhot A, et al. Evaluation of an alternative chest physiotherapy method in infants with respiratory 
syncytial virus bronchiolitis. Respir Care. 2011;56:989-94. PMid:21352671. http://dx.doi.org/10.4187/respcare.00721

27. Sánchez Bayle M, Martín Martín R, Cano Fernández J, Martínez Sánchez G, Gómez Martín J, Yep Chullen $\mathrm{G}$, et al. Chest physiotherapy and bronchiolitis in the hospitalised infant. Double-blind clinical trial. An Pediatr (Barc). 2012;77:5-11. PMid:22281403. http://dx.doi. org/10.1016/j.anpedi.2011.11.026

28. Gomes EL, Postiaux G, Medeiros DR, Monteiro KK, Sampaio LM, Costa D. Chest physical therapy is effective in reducing the clinical score in bronchiolitis: randomized controlled trial. Rev Bras Fisioter. 2012;16:2417. PMid:22499404. http://dx.doi.org/10.1590/ S1413-35552012005000018

\section{Correspondence}

\section{Hugo C. D. Souza}

Universidade de São Paulo

Faculdade de Medicina de Ribeirão Preto

Departamento de Biomecânica, Medicina e Reabilitação

Av. Bandeirantes, 3900

CEP 14049-900, Ribeirão Preto, SP, Brasil

e-mail: hugocds@fmrp.usp.br 